\title{
PULSE WAVE VARIABILITY WITHIN TWO SHORT-TERM MEASUREMENTS
}

\author{
David Korpas, Jan Halek
}

\author{
Department of Medical Biophysics, Faculty of Medicine, Palacky University, Olomouc \\ e-mail:david.korpas@seznam.cz
}

Received: September 11, 2006; Accepted: November 11, 2006

Key words: Pulse wave/Blood flow/Plethysmography/Arterial elasticity/Blood volume/Measurement techniques

Background: Pulse wave analysis permits non-invasive assessment of indices of arterial elasticity. Decreased arterial elasticity results in higher pulse wave velocity and therefore earlier wave reflection.

Aim: The aim of this study was to evaluate the short-term variability for measurement of surface arteries.

Method: For detection of artery wall movement, a plethysmography method was used. The changes in artery volume, affecting the fluid behind the scanning membrane, transfer themselves into pressure changes and move towards the positive input of a sensitive differential pressure transducer. The output is carried to a notebook computer and analysed within the frequency and time domain. To correlate arterial pulse waves, the following parameters were used: relative crest time RCT, interwave distance IWD, dicrotic wave amplitude DWA and dicrotic wave time DWT. These parameters were analysed using Bland-Altman plots.

Results: Two data points $(5.6 \%)$ fall outside the $2 *$ SD range for both IWD and DWA parameters on posterior tibial artery and one data point $(2.8 \%)$ for DWT. Three data points $(8.3 \%)$ fall outside the $2 *$ SD range for IWD and two data points for both DWA and DWT on the radial artery. Two data points fall outside the RCT measured on the ulnar artery, and all but one for IWD, DWA and DWT lie within the $2 *$ SD range.

Conclusions: Pulse wave analysis showed reproducibility in a short-term study of all three measured distal arteries and is therefore suitable for use in patients for observing the progression of artery-wall-involving diseases.

\section{INTRODUCTION}

Increased arterial stiffness is an important marker of atherosclerosis, which is the main cause of cardiovascular mortality in developed countries. During the life, the artery wall constantly loses elasticity and becomes rigid $^{6,8,13}$. Hypertension and other diseases like diabetes mellitus accelerate this process ${ }^{3,8}$. The increase in artery wall stiffness is noticeable from the beginning of the atherosclerosis process, before anatomical changes and clinical manifestations are observed. Some methods for artery stiffness measurement are based on measurement of pulse wave velocity and analysis of pulse wave curve form ${ }^{9,11,14}$.

The pulse wave, manifested as artery wall movement, is a complex physiological phenomenon, observable and measurable in an arterial system during blood circulation. During the heart systole, a certain blood volume is expelled and it propagates through arteries due to the reciprocal transformation between kinetic energy of a segment of the expelled blood volume and the potential energy of a stretched segment of the resilient vascular wall. In each region through which the pulse wave runs, pressure changes, blood flow velocity changes and profile changes can be observed as well. In this way, an instrument for detection of artery profile changes enables us to image the pulse wave time progress of a chosen artery, which is compressible from the body surface.
There are several methods of non-invasive pulse wave measurements available based on different principles. First to be used clinically was Doppler ultrasound. Transparent and reflex photoplethysmography methods are also often used $^{1,2,4,10}$. There are also methods based on direct measurement of volume changes using pressure transducers with inductive or capacity probes ${ }^{12}$.

The physics of blood circulation in arteries can be demonstrated on model mechanical system. This system is composed of a piston and elastic hose, which is filled with fluid and terminated by wave resistance. The piston (model of heart) injects a certain volume of fluid (blood) into the hose (model of arteries). If we have a rigid hose, the whole volume of fluid would move, but if we have an elastic-walled-hose, only the fluid at the beginning of the hose moves. The pressure then increases, which causes local widening of the hose and local accumulation of the fluid. Tension of the widened elastic wall causes contraction and pushes the fluid to the next part of the hose. The hose widens there again and the pressure increases. The process continues along the whole length of the hose. The process, which we have just described, runs continuously as a propagating pulse wave along the hose. In every region where the pulse wave runs, three related effects can be observed: liquid flow (flow wave), a pressure change (pressure wave), and a cross-section change (volume wave). It is the artery volume change that our method utilizes. 


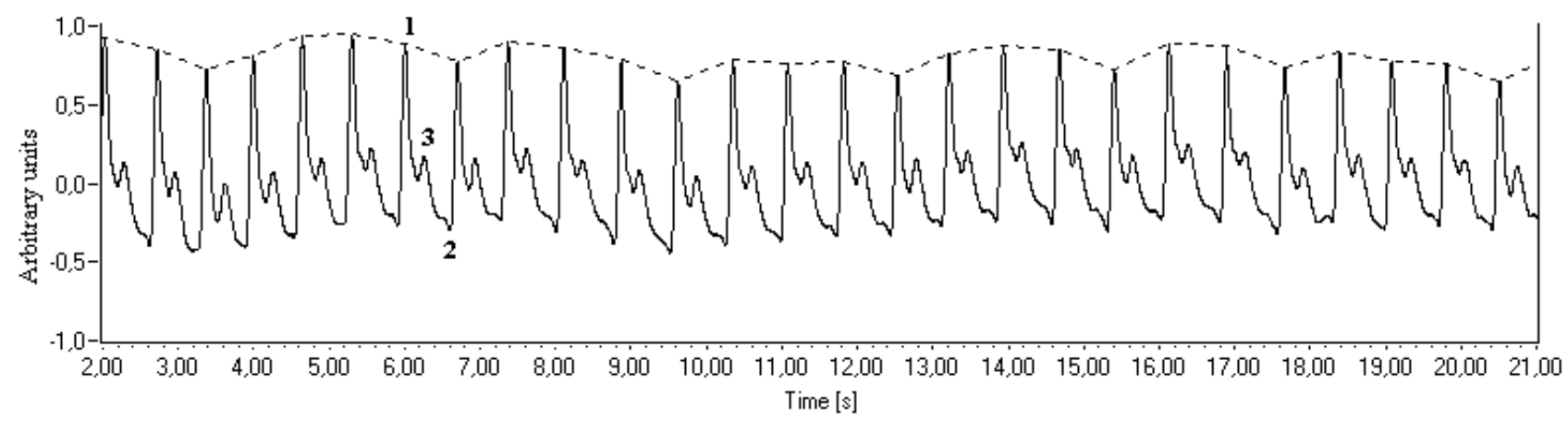

Fig. 1. A fragment of recorded pulse wave of radial artery, measured by the device, with main features. Systole peaks (1), diastole peaks (2), dicrotic peaks (3), respiratory waves (broken line).

The forward wave spreads to the periphery, where it does not dissipate, but is reflected back to central vascular system. This retrograde propagation causes an increase of blood pressure again. Standing waves arise. The resulting pulse waveform is determinated by phase sum of forward and reflected waves. The time differences of reflected pulse wave in each part of circulation are responsible for form differences of central and peripheral pulse wave.

We measured the volume pulse wave of three important arteries: posterior tibial, ulnar and radial artery, but the method is sensitive enough to be used for measurements on other arteries too, like superficial temporal artery, dorsalis pedis artery, carrotis arteries or brachial artery in their appropriate palpation spots.

On Fig. 1, typical pulse wave run is shown. For careful evaluation, a low frequency rhythm like respiratory waves is important.

On the pulse wave, several main features (Fig. 2) can be observed. There are systole peaks (1), diastole peaks (2) and dicrotic peaks (3), caused by standing waves. There are also some low frequency waves, e.g. respiratory waves (broken line). In the waveform, we can measure a number of parameters. Some of them are shown in Fig. 2.

For evaluation of the pulse wave, the four following parameters were chosen: Relative crest time RCT $=\mathrm{TP} 1 /$ TPT; Interwave distance IWD = IWT / TPT; Dicrotic wave amplitude DWA = AP2 / AP1; Dicrotic wave time DWT $=$ TP2 $/$ TPT. These are derived from the rate of basic distances in Fig. 2. All these parameters are results of times intervals or amplitudes divisions and therefore are unit-free.

\section{MATERIALS AND METHODS}

\section{Technical description of the device}

The general chart of the whole device is shown in the Fig. 3. It consists of a scanning membrane on a probe, a surface stabiliser, a probe, a liquid and air part for pressure transmission, and a differential pressure scanner. Further there is a connecting block, a measuring card

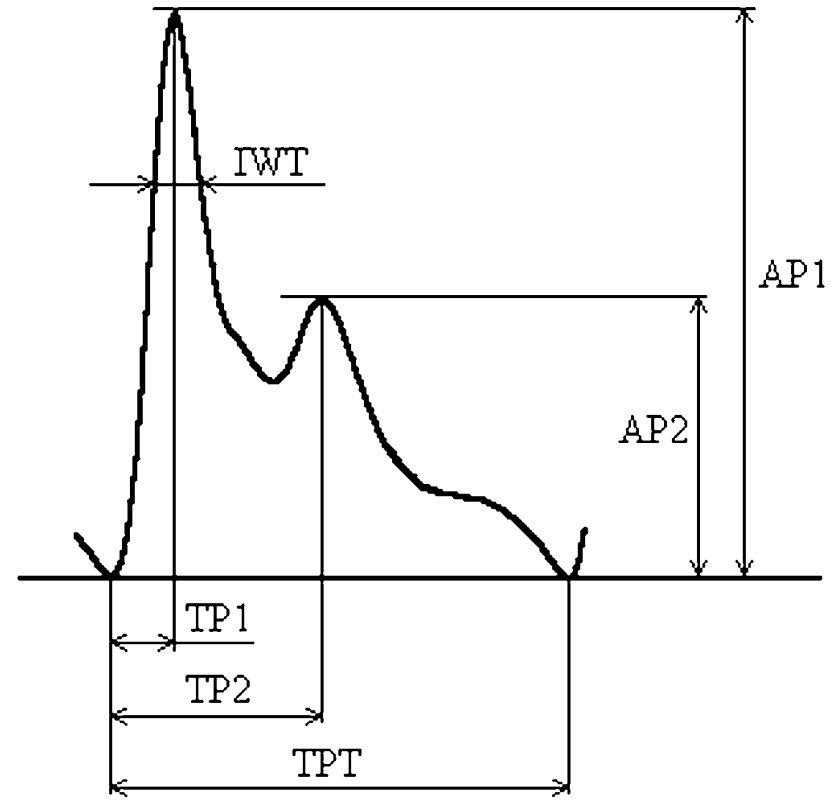

Fig. 2. Description of pulse wave parameters: (TP1) Time to systolic peak, (TP2) Time to dicrotic peak, (TPT) Total pulse time, (IWT) Interwave time in 2/3 of the peak height, (AP1) Amplitude of systolic peak, (AP2) Amplitude of dicrotic peak.

and a PC with software for the analysis and storage of volume pulse waveforms?

Basically there are two parts. First there is a mechanical plethysmography part, which converts the volume changes of the artery into an electrical voltage. Next there is an electrical computer part, which processes the electric signal, displays, saves and analyses the curves. A special section is a stabilisation system for fixation of the probe to the limb, or another part of the body suitable for measurement.

The volume changes, caused by spreading pulse wave during heart systole are scanned through the probe. As a matter of fact, these volumes changes affect the uncompressed fluid behind the membrane on the probe, transfer themselves into pressure changes and move through the 

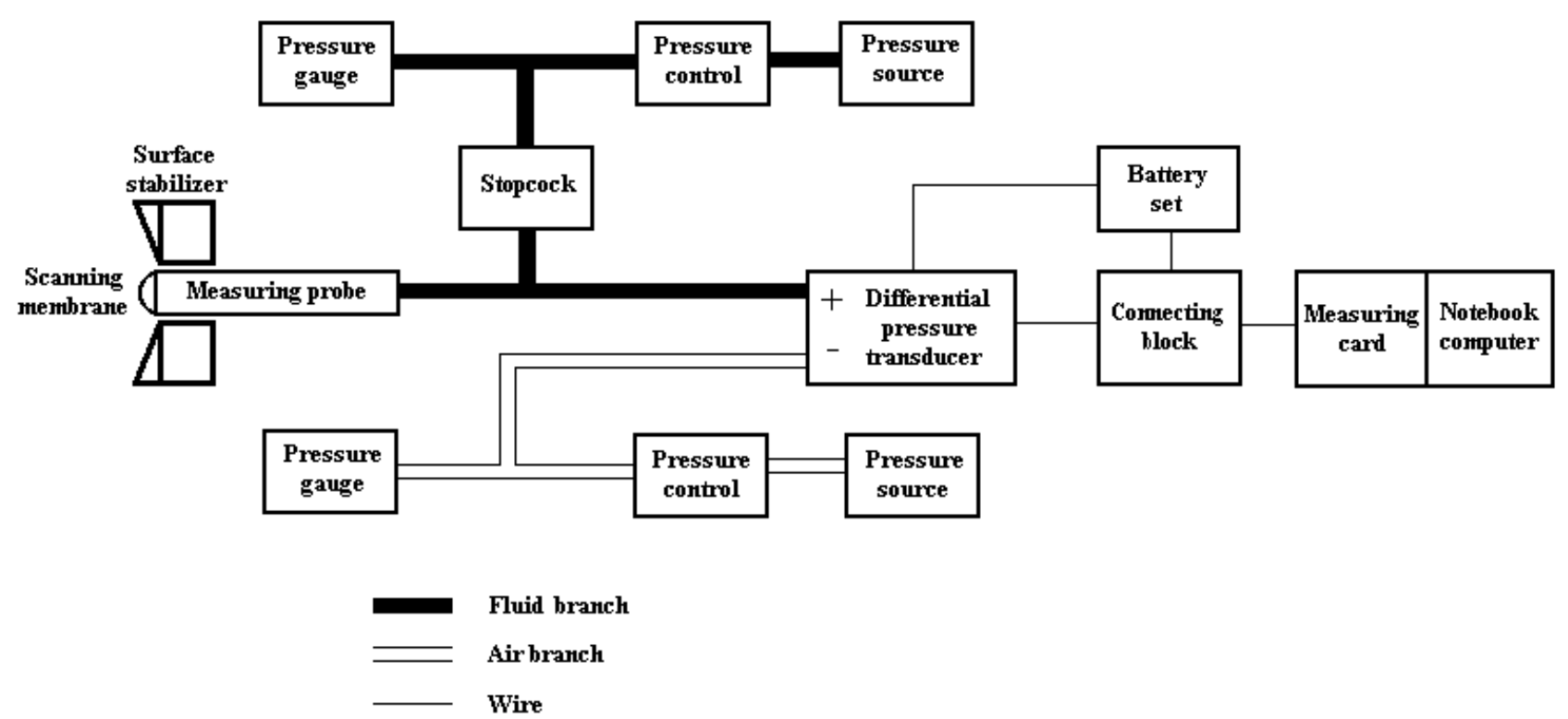

Fig. 3. A general chart of the device.

system of thin towards the positive input of a very sensitive differential pressure transducer. The probe membrane has a convex form because of an inside pressure of the fluid media. This inside pressure is caused by the fluid pressure source and regulated with pressure control and gauge. Its value, approximately $12 \mathrm{kPa}$, is determinated by mechanical properties of the membrane. The probe is made of brass, its in-diameter (membrane) is $8 \mathrm{~mm}$ and its out-diameter (hose) is $2 \mathrm{~mm}$. This tapering is necessary for obtaining the maximum output signal. Directly on the probe, independent of the main stabilisation system, there are small jaws, preventing random movements. A stopcock is used for separation of the pressure source, not to diminish the measured pulsation.

A compensation air branch is connected to the negative input of the differential pressure transducer. This branch consists of pressure source, pressure control and gauge. It allows regulation of the compensation pressure in a range from 0 to $40 \mathrm{kPa}$. The air branch allows precise control of static pressure for the differential pressure transducer and therefore the balancing and adjusting of the correct pressure to the pulse point. This compensation pressure is equal to the addition of contact pressure of the probe to the artery and inside pressure of the fluid in the liquid branch.

An essential part of the device is the part for locating the membrane position on the probe in relation to the location on the artery. It is necessary to eliminate any deliberate, as well as involuntary movement towards the membrane. This is provided by a stabilisation system. It consists of a holder and a probe-carrier, which is flexibly connected to the holder and allows variability of probe positioning towards the artery.

The curves according to Fig. 2 can be calibrated to approximate blood pressure using the tonometry cuff and associate the systole pressure to systolic peaks a diastolic pressure to diastolic valleys.

\section{Measurement procedure}

Thirty-six subjects ( 9 men and 27 women), aged 1828 , mean age 22 years, recruited from university staff and students, were examined. They had no previous cardiovascular of other serious disease and used no cardiovascular medication. Each subject lay in a supine position for 5 minutes in a quiet room before the pulse wave and blood pressure recordings were taken. Two measurement were recorded on two occasions with a time interval of between 3 and 11 hours (median 7 hours). We used the posterior tibial, radial and ulnar artery for the measurement. The measurement probe was placed on the artery palpation spot and positioned carefully until the signal was strong enough. After that the computer recorded the signal for 30 seconds. We used the mean of all pulse waves under a respiratory wave as a valid value for each subject.

\section{Data analysis}

All data were analysed as means \pm standard deviation (SD). The Bland - Altman plots were used for limits for agreement of RCT, IWD, DWA and DWT ${ }^{2}$. The means of the dependent variables are plotted against the differences in means, and limits of agreements are within $2 * \mathrm{SD}$ of the difference in means.

\section{RESULTS}

\section{Quality of measured curves}

Producing the best possible quality of measured waves were the basic condition for the design. Owing to the mechanical principle of the plethysmography part, all random movements between artery and probe must be eliminated. Therefore using of a solid holder, designed specially for each artery, is necessary. 

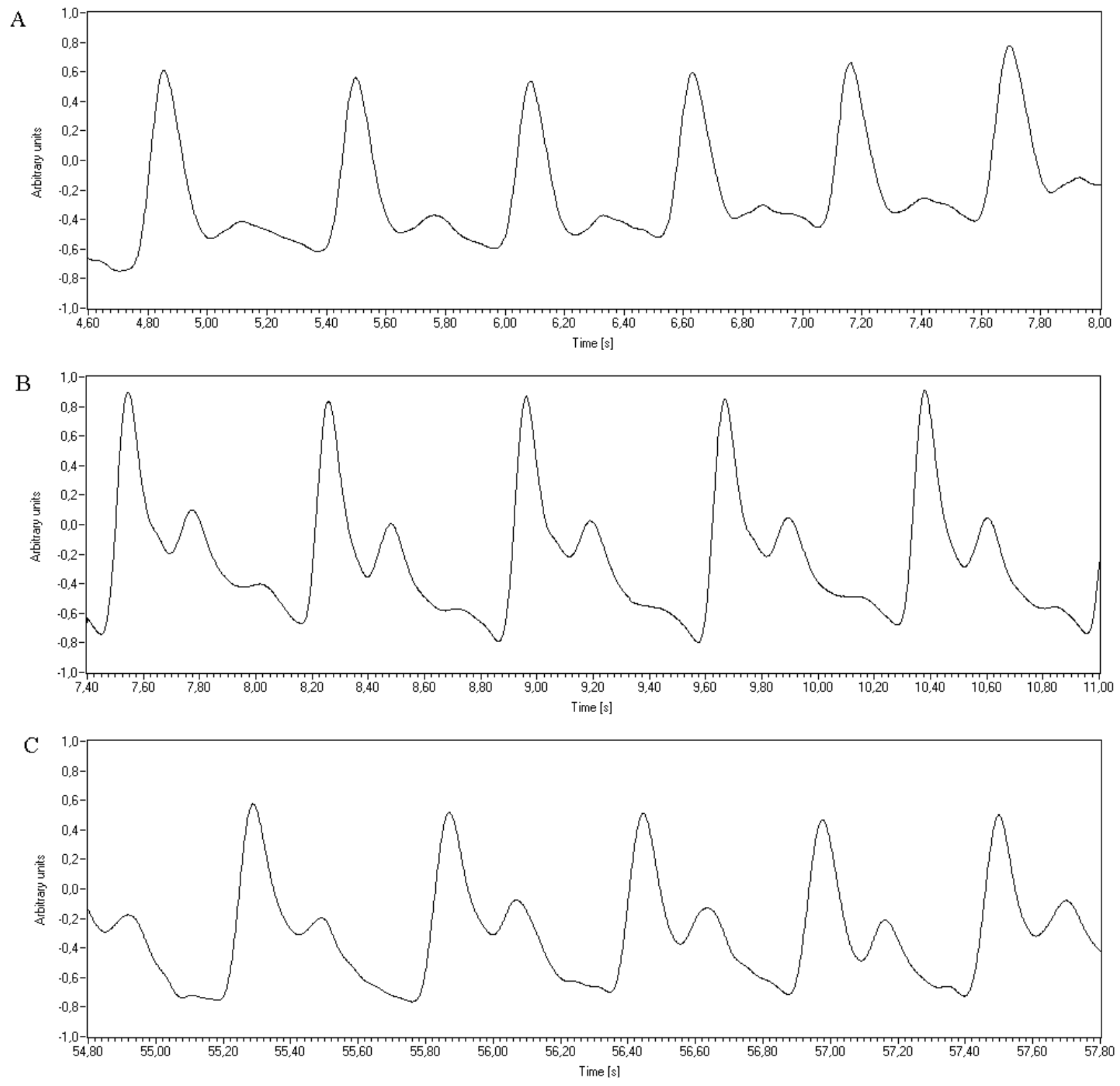

Fig. 4. The quality of waveforms measured on three arteries: (A) Posterior tibial artery, (B) Radial artery, (C) Ulnar artery.

\section{Short-term measurement}

Fig. 5, Fig. 6 and Fig. 7 show the Bland-Altman plots of the mean differences between two measurements plotted against the mean values of these two measurements on three arteries.

The mean differences for posterior tibial artery were: $-0.0019 \pm 0.0100$ for RCT, $0.0003 \pm 0.0124$ for IWD, $-0.0034 \pm 0.0330$ for DWA and $-0.0017 \pm 0.0220$ for DWT. The mean differences for radial artery were: -0.0018 \pm 0.0260 for RCT, $0.0013 \pm 0.0110$ for IWD, $-0.0077 \pm$ 0.0751 for DWA and $0.0000 \pm 0.0588$ for DWT. The mean differences for ulnar artery were: $0.0000 \pm 0.0096$ for RCT, $0.0018 \pm 0.0123$ for IWD, $0.0026 \pm 0.0397$ for DWA and $-0.0014 \pm 0.0225$ for DWT.
Two data points $(5.6 \%)$ fall outside the $2 *$ SD range for both IWD and DWA parameters on posterior tibial artery and one data point $(2.8 \%)$ for DWT. Three data points $(8.3 \%)$ fall outside the $2 *$ SD range for IWD and two data points for both DWA and DWT on the radial artery. Two data points fall outside for RCT measured on the ulnar artery, and all but one for IWD, DWA and DWT lie within the $2 * \mathrm{SD}$ range.

\section{DISCUSSION}

The main arteries pulse wave analysis is a possible access in the diagnostic of the vascular diseases, such as artery seals or atherosclerosis. Non invasive pulse wave es- 

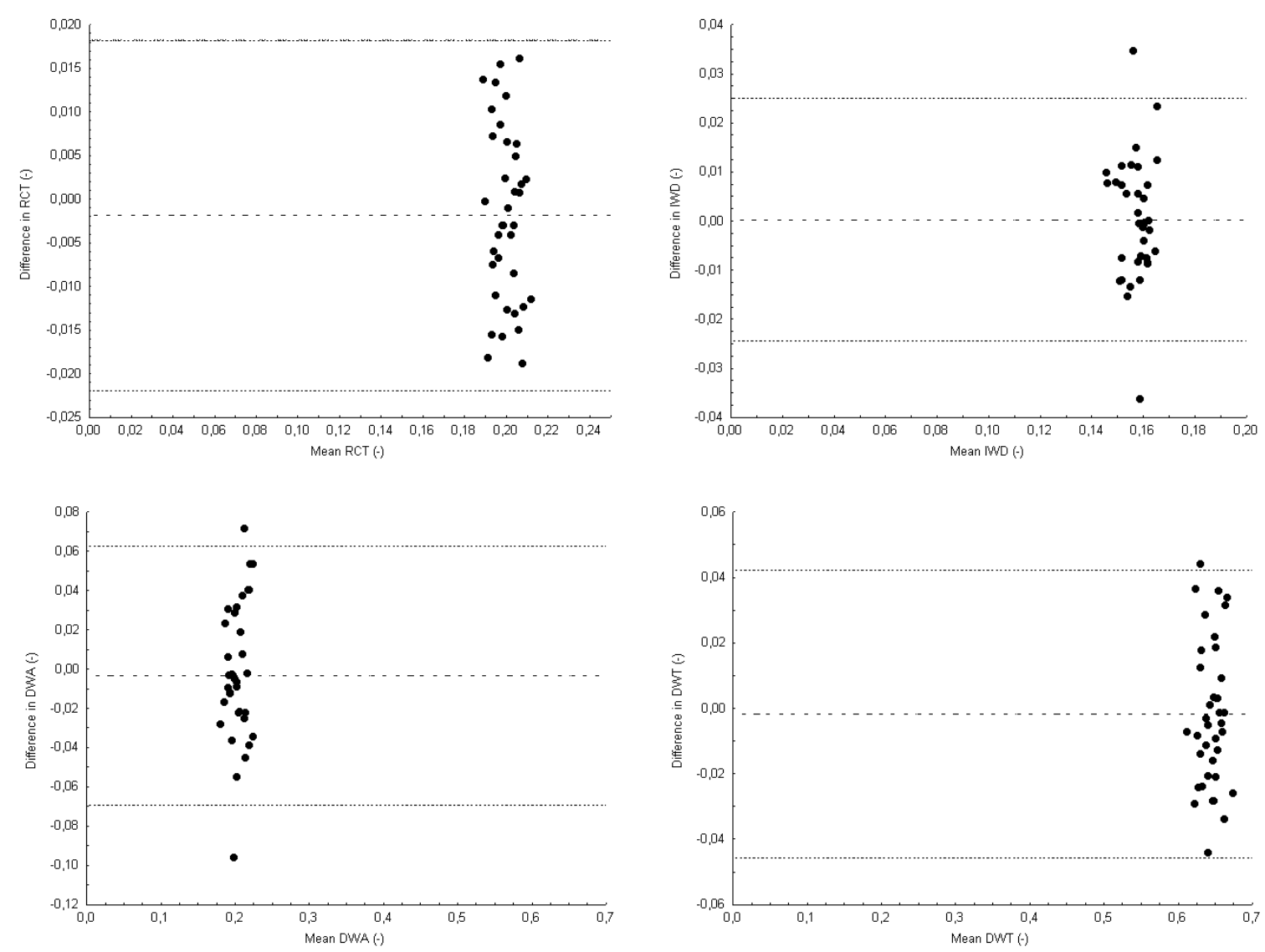

Fig. 5. Bland-Altman plot $(n=36)$ to show short-term reproducibility on posterior tibial artery. The broken line is the mean and dotted lines are mean $\pm 2 *$ SD.
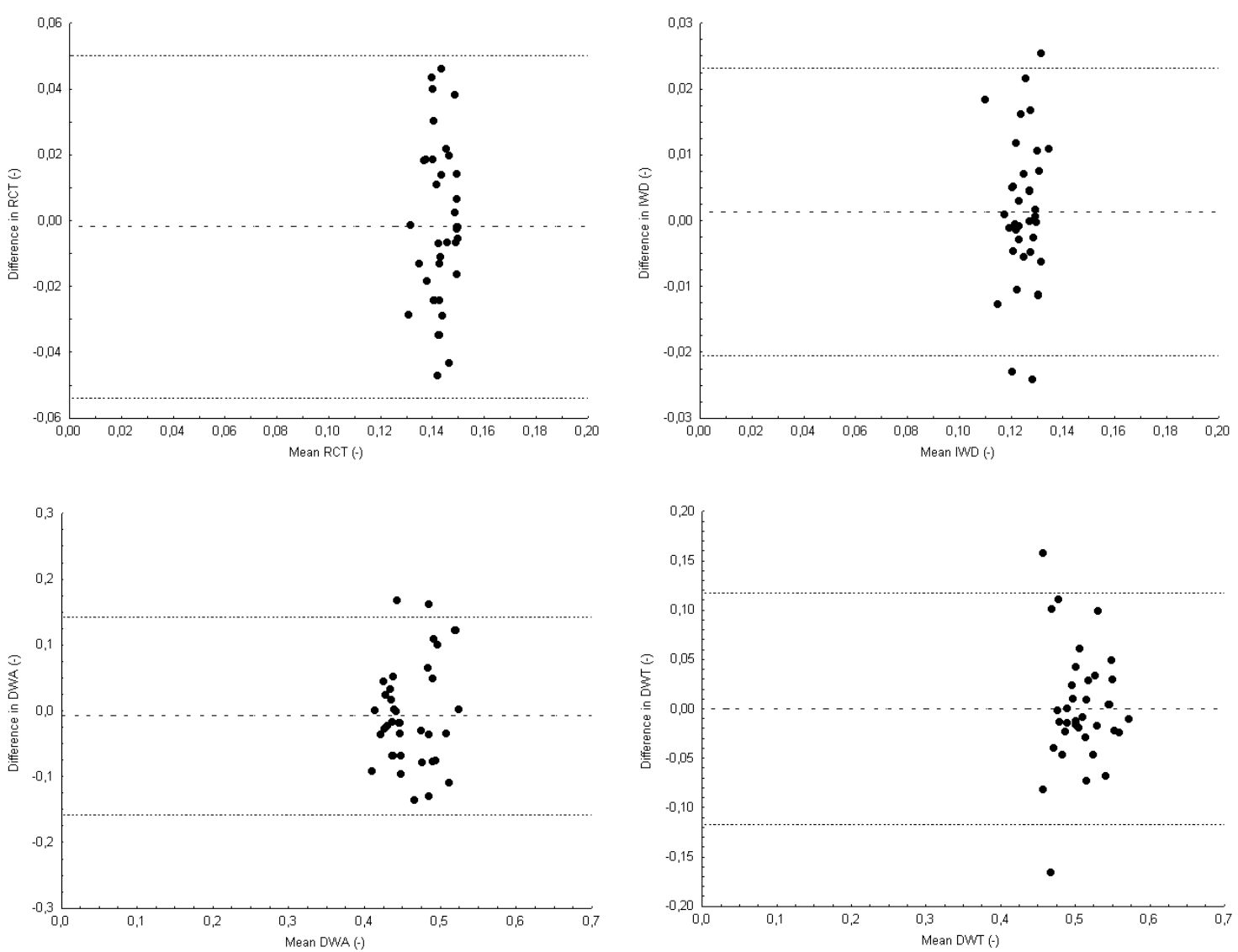

Fig. 6. Bland-Altman plot $(n=36)$ to show short-term reproducibility on radial artery. The broken line is the mean and dotted lines are mean $\pm 2 *$ SD. 

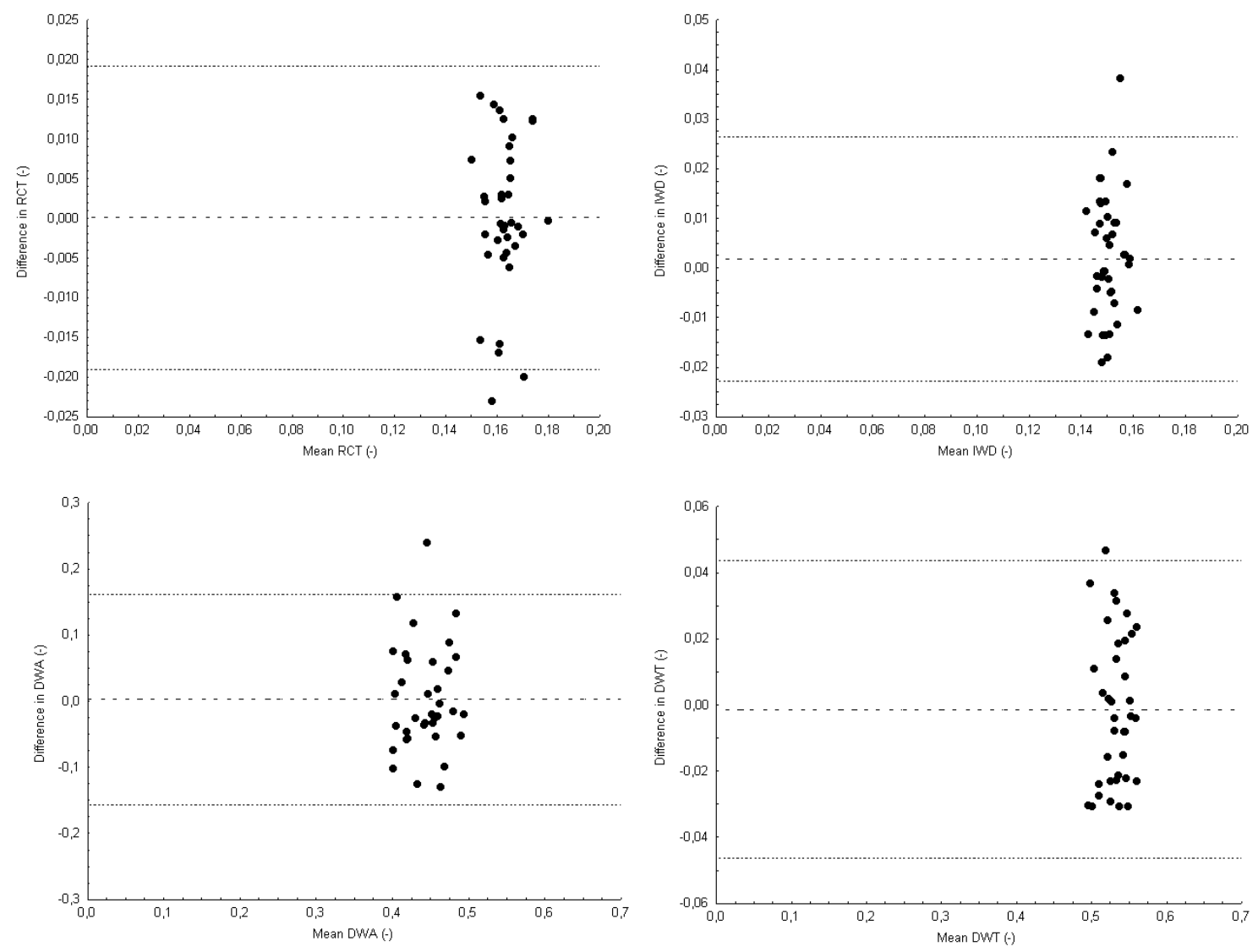

Fig. 7. Bland-Altman plot $(n=36)$ to show short-term reproducibility on ulnar artery. The broken line is the mean and dotted lines are mean $\pm 2 *$ SD.

timation by means of plethysmography methods provides valuable information on arteries. The properties of the artery wall like its elasticity, thickness and atherosclerotic changes may cause disturbance in blood flow and therefore in pulse wave curve.

In our study, we evaluated the pulse wave according to four parameters: Relative crest time RCT, interwave distance IWD, dicrotic wave amplitude DWA and dicrotic wave time DWT.

Our results show, that $\mathrm{PW}$ analysis of main surface arteries is reproducible for short-term assessment. Whereas similar reproducibility studies have been done for patient with diabetes, hypertension and renal failure on radial artery or computed aortal wave, the present paper confirms the reproducibility in ulnar and tibial posterior artery, as well.

\section{REFERENCES}

1. Blanc VF, Haig M, Troli M, Sauve B. Computerised photoplethysmography of the finger. Can J Aneasth 1993; 40:271-278.

2. Bland JM, Altman DG Statistical methods for assessing agreement between two methods of clinical measurement. Lancet 1986; 8:307-310

3. Brooks B, Molyneaux L, Yue DK. Augmentation of central arterial pressure in type I diabetes mellitus. Diabetes Care 1999; 22:1722-1727.
4. Filipovsky J, Svobodova V, Pecen L. Reproducibility of radial pulse wave analysis in healthy subjects. J Hypertens 2000; 18:10331040.

5. Guyton AC, Hall JE. Textbook of medical physiology. Guyton $9^{\text {th }}$ Edition, 1996.

6. Kelly R, Hayward C, Avolio A, O’Rourke M. Noninvasive determination of age-related changes in the human arterial pulse. Circulation 1989; 80:1652-1659.

7. Korpas D, Hálek J. A device for non-invasive pulse wave measurement [Patent description], Prague: Czech patent office, 2003.

8. Nichols WW, O'Rourke FM. McDonalds boold flow in arteries $4^{\text {th }}$ edition. London: Arnold, 1998.

9. Oliva I, Roztočil K. Pulse wave in diagnoses of lower limb ischemia disease. Prague: Avicenum, 1982.

10. Peňáz J. A device for automatic non-invasive continuous measurement of blood pressure [Patent description], Prague: Czech patent office, 1989.

11. Savage MT, Ferro CJ, Pinder JS. Reproducibility of derived central arterial waveforms in patients with chronic renal failure. Clinical Science 2002; 103:59-65.

12. Schurmann M, Zaspel J, Gradl G. Assessment of the peripheral microcirculation isung computer-assisted venous congestion plethysmographyin post-traumatic complex regional pain syndrome type I. J Vasc Res 2001; 38:453-461.

13. Vaitkevicius PV, Fleg JL, Engel JH, O’Connor FC, Wright JG, Lakatta LE, Yin FC. Effects of age and aerobic capacity on arterial stiffness in healthy adults. Circulation 1993; 88:1456-1462.

14. Wilkinson IB, MacCallum H, Flint L, Cockcroft JR, Newby DE, Webb DJ. The influence of heart rate on augmentation index and central arterial pressure in humans. J Physiol 2000; 525:263270. 